\title{
Quaternary Structure of the European Spiny Lobster (Palinurus elephas) $1 \times 6$-mer Hemocyanin from cryoEM and Amino Acid Sequence Data
}

\author{
Ulrich Meissner $^{1 *}$, Michael Stohr ${ }^{1}$, Kristina Kusche ${ }^{1}$ \\ Thorsten Burmester $^{1}$, Holger Stark ${ }^{2}$, J. Robin Harris ${ }^{1}$, Elena V. Orlova ${ }^{3}$ \\ and Jürgen Markl ${ }^{1}$
}

${ }^{1}$ Institute of Zoology

University of Mainz

Muellerweg 6, D-55099 Mainz

Germany

${ }^{2} \mathrm{MPI}$ for Biophysical Chemistry

Am Fassberg 11, D-37077

Göttingen, Germany

${ }^{3}$ Department of

Crystallography, Birkbeck

College, University of London

Malet Street, London WC1E

7HX, UK

\begin{abstract}
Arthropod hemocyanins are large respiratory proteins that are composed of up to 48 subunits $(8 \times 6$-mer $)$ in the $75 \mathrm{kDa}$ range. A 3D reconstruction of the $1 \times 6$-mer hemocyanin from the European spiny lobster Palinurus elephas has been performed from 9970 single particles using cryoelectron microscopy. An $8 \AA$ resolution of the hemocyanin $3 \mathrm{D}$ reconstruction has been obtained from about 600 final class averages. Visualisation of structural elements such as $\alpha$-helices has been achieved. An amino acid sequence alignment shows the high sequence identity $(>80 \%)$ of the hemocyanin subunits from the European spiny lobster P. elephas and the American spiny lobster Panulirus interruptus. Comparison of the P. elephas hemocyanin electron microscopy (EM) density map with the known $P$. interruptus $\mathrm{X}$-ray structure shows a close structural correlation, demonstrating the reliability of both methods for reconstructing proteins. By molecular modelling, we have found the putative locations for the amino acid sequence (597-605) and the C-terminal end (654-657), which are absent in the available $P$. interruptus X-ray data.
\end{abstract}

(C) 2002 Elsevier Science Ltd. All rights reserved

Keywords: 3D-EM, cryoelectron microscopy (cryoEM); 3D reconstruction; Crustacea; hemocyanin; quaternary structure depending on the species). Within the native Hc the subunit composition may be heterogeneous and in several Hc multimers-specific structural roles have been attributed to different subunits. ${ }^{1,4}$ The structural hierarchy of arthropod hemocyanins, as assemblies of an increasing number of $1 \times 6$-mers, has been correlated with functional cooperativity during oxygen binding. ${ }^{5}$ This can best be described by the nesting model. $6,7,8$ Arthropod hemocyanins achieve Hill coefficients (a measure of cooperativity) of up to ten and therefore provide useful molecular models for the study of structure-function relationships of high molecular mass oligomeric proteins. ${ }^{1,2}$

In recent years, many arthropod hemocyanin sequences have been determined and compared,,, 10 and X-ray structures of hemocyanin subunits from a chelicerate (the horse shoe crab Limulus polyphemus) and a crustacean (the spiny lobster Panulirus interruptus) are available. ${ }^{11,12}$ The understanding of conformational changes during cooperative interactions requires knowledge of the high-resolution quaternary structure rather than 
100 PelHC2 DVHSSDNAHKOODVNHLLDKIYEPI KDEELLHNAOTFDPVADTS IYVDDGAAAKTLMOELNDHRLLEOHHWFSLFNTROREEALMLFTVLNQCKNWNCFR PelHC3 DVHSSDNAHKOODVNHLLDKIYEPIKDEKLHNTAOTFDPVADTSIYGDDGAAAKTLMOELNDHRLLEQHHWFSLFNTRQREEALMLFTVLNQCKDWNCFL PinHCa DALGTGNAOKOQD INHLLDKIYEPTKYPDLKDIAENFNPLGDTSIYNDHGAAVETLMKELNDHRLLEQRHWYSLFNTRQRKEALMLFAVLNQCKEWYCFR PinHcb DALGTGNANKOQDINHLLDKIYEPT KY PDLKDIAENFNPLGDTSIYNDHGAAAEALMKELNDHRLLEQRHWFSLFNTRQREEALMLFAVLNQCKEWYCFR

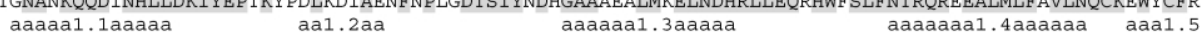
CHO

PeIHC1 NNAAFFRERMNEGEFVYALYVSVIHSKLGDGIVLPPLYEVTPHMFTNSEVIDKAYSAKMTHKEGTFNMSFTGTKKNKEORVAYFGEDIGMNIH HVTWFIM PelHC2 NNAAFFRERMNEGEFVYALYVSVIHSKLGDGVVLPLYEVTPHMFTNNEVIDKAYSAKMTHEGNTFDMSFTGTKKNREQRVAYFGEDIGMNIHHVTWHMD
PelHC3 NNAAFFRERMNEGEFVYALYVSVIHSKLGDGIVLPPLYEVTPHMFTNNEVIDKAYSAKMTHKEGTFNMSFTGTKKNKEQRVAYFGEDIGMNIHHVTWHMD PelHC3 NNAAFFRERMNEGEFVYALYVSVIHSKLGDGIVLPPLYEVTPHMFTNNEVIDKAYSAKMTHKEGTFNMSFTGTKKNKEQRVAYFGEDIGMNIHHVTWHMD
PinHCa SNAAYFRERMNEGEFVYALYVSVIHSKLGDGIVLPPLYEITPHMFTNSEVIDKAYSAKMTQKPGTFNVSFTGTKKNREQRVAYFGEDIGMNIHHVTWHMD PinHcb SNAAYFRERMNEGEFVYALYVSVIHSKLGDGIVLPPLYEITPHMFTNSEVIDKAYSAKMTQKPGTFNVSFTGTKKNREQRVAYFGEDIGMNIHHVTWHMD aaaaa aaal.6aaa bbblabbbb aaaal.7aaaa bbb1Bbbb aaaaa2.1aaaa

TTARFDAERLSNWMEPVDELHWDDI IHEGFAPHTSYKYGGEFPVRPDNIRFEDVDGVAHVHDMEITEVRIRDI PelHC2 FPFWWQDSYGYHLDRKGELFFWVHHQLTARFDAERLSNWMDPVDELHWEEVIHEGFAPHASYKYGGEFPTRPDNTQFKNVDGVARVRDMEITESRIRDAI PelHC3 FPFWWDDSYGYHLDRKGELFFWVHHQLTARFDAERLSNWLDPVAELHWVDVIHEGFAPHASYKHGGEFPTRPDNIRFKNVDGVARVHDMEITESRIRDAI PinHCa FPFWWEDSYGYHLDRKGELFFWVHHQLTARFDFERLSNWLDPVDELHWDRIIREGFAPLTSYKYGGEFPVRPDNIHFEDVDGVAHVHDLEITESRIHEAI PinHc $b$ FPTWWQDSYGYHLDRKGELFFWVHHQLTARFDFERLSNWLDPVDELHWDRIIREGFAPLTSYKYGGEFPVRPDNIHFEDVDGVAHVHDLEITESRIHDAI aa aaaaaaaaa2,2aaaaaaaa bb2Abb bbbbb2Bbbbb bbbbb2Cbbbbb aaaaaa2.3aaa

Pe1HC1 AHGYITATDGHTIDIROPNGIELLGDIIESSMYSSNPHYYGSI HNAMLGROGDPHGKFDMPPGVMEHFETATRDPSFFRIHKYMDNIFKEHTDSFPD PelHc2 AHGYITATDGHTIDIROPNGIELLGDIIESSMYSSNPOYYGSLHNTAHMMLGROGDPHGKFDMPPGVMEHFETATRDPSFFRLHKYMDNIFKEHTDSFP PelHc3 AHGYITATDGHTIDIROPNGI LLLGDI IESSMYSSNPHYYGSI HNTAHMMLGROGD PHGKFNMPPGVMEHFETATRDPSFFRLHKYMDNIFKEHTDSFP PinHCa DHGYII PINH

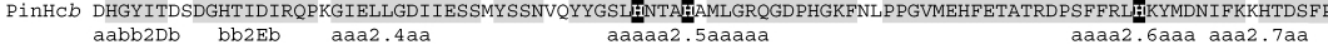

Pe1HC1 YTHEDLEFGVSVDNTATDGHLTTEFDOFKYSLVNAVDSGENVEDVATYANVHRLNHEEFTYETEVRNHNE-EKFATVRIFL S- 50 PelHC1 YTHEDLEF PGVSVDNIAIDGHLTTFFDQFKYSLVNAVDSGENVEDVAIYANVHRLNHEEFTYEIEVRNHNE-EKFATVRIFLCPTEDNNGITLNLDEER PelHC2 YTHEDLEFPGVSVENIAIDGHLTTFFDQFKYSLVNAVDSGENIEDVEIYANVHRLNHEEFTYVIEVSNANE-VHLATVRIFLCPTEDNNGITLNLDEER PeIHC3 YTHEDLEFAGVSVDNIAIDGHLTTFFDQFKYSLVNAVDSGENVEDVAIYANVHRLNHEEFTYDIEVRNHNEEEKFATVRIFLCPTEDNNGITLNLDEAR PInACa YTDNLEF PinHcb YTHDDLEFAGMVVDGIAIDGELITFFDEFQYSILNAVDSGENIEDVDINARVHRLNHNEFTYKITMSNNNDGERLATFRIFLCPIEDNNGITLTLDEARV aa3.1a bbbbbbb3Abbbbbb b3Bb bbb3Cbbb bbbbbbb3Dbbbbbb bbbbbb3Ebbbbbb 3Fb aa3.2a

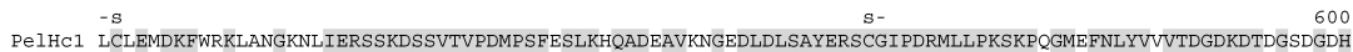
Pe1HC2 LCLELDKFWTKLANGKNLIERSSKDSSVTVPDMPSFESLKHOADEAVKNGEDLDLSAYERSCGI PDRMLLPKSKPQGMEFNLYVAVTDGDKDTDGSDGDH Pe1HC3 LCLEMDKFWKKLGNGLNMIERSSKDSSVTVPDMPSFESLKHQADEAVKNGEDLDLSAYERSCGIPDRMLLPKSKPQGMEFNLYVAVTDGDKDTDGSDGDH PinHCa FCIELDKFFQKVPSGPETIERSSKDSSVTVPDMPSFQSLKEQADNAVNGGHDLDLSAYERSCGI PDRMLLPKSKPEGMEFNLYVAVTDGDKDTEGHNGGH PinHcb FCIELDKFFQKVPSGPETIERSSKDSSVTVPDMPSFQSLKEQADNAVNGGHDLDLSAYERSCGIPDRMLLPKSKPEGMKFNLYVAVTDGDKDTEGHNGGH a bbbb3Gbbbb bbbb3Hbbbb bbb3Ibbbaaaa3.3aaaa

PEIHC1 DHHGTHAQCGIHGEQYPDHRPLGYPLERRIPDERVFDGVSNIKHALVKIVHDPELRA 656 PelHC2 DHHGTHAOCGIHGEOYPDHRPLGYPLERRIPDERVFDGVSNIKHALVKIVHDPELRA 656 PElHC3 DHHGTHAQCGIHGEQYPDHRPLGYPLERRIPDERVFDGVSNIKHALVKIVHDPELRA 657 PinHCa DYGGTHAQCGVHGEAYPDNRPLGYPLERRIPDERVIDGVSNIKHVVVKIVHHLEHHD 657

PINHCb DYGGTHAQCGVHGEAFPDNRPLGY PLERRI PDERVIDGVSNIKHVVVKIVHHLEHHD $65^{\circ}$ $* * * * * \quad \mathrm{bbb} 3 \mathrm{Kbbb}$

Figure 1. Sequence alignment of spiny lobster hemocyanin subunits. PelHc1 to PelHc3, the P. elephas hemocyanin subunits 1-3 ( $\alpha$-type); PinHca and PinHcb, the P. interruptus hemocyanin subunits $a$ and $b$ (both $\alpha$-type). The conserved residues are shaded in grey, the copper-binding histidine residues are white on a black background. The secondary structure elements are displayed as derived from the PinHc. ${ }^{11,52} \mathrm{a}, \alpha$-helices; $b, \beta$-sheets; $*$, residues not included in PinHc PDB-file; ${ }^{11} \mathrm{~s}$, disulphide bridges; $\mathrm{CHO}$, carbohydrate. The $\gamma$-type subunit of PinHc is not displayed.

that of subunit arrangement alone. This has been provided by X-ray crystallography for $P$. interruptus hemocyanin (PinHc).$^{11,13,14}$ However, the Hc $1 \times 6$-mer analysed in these studies was not the native molecule that contains three different subunit types, but an in vitro reassembly product containing equal quantities of subunits $a$ and $b$. Moreover, high resolution X-ray data of the deoxygenated $1 \times 6$-mer is still lacking, and all attempts to crystallize arthropod hemocyanin multimers have failed. Electron microscopy (EM), as an alternative approach, has produced low resolution quaternary structures, ${ }^{15-21}$ but no highresolution (sub-10 $\AA$ ) cryoEM data set of arthropod hemocyanin has been published to date.

CryoEM followed by single particle analysis has become a standard method for determining molecular structure at medium resolution. ${ }^{22}$ For several molecules, 3D reconstruction from EM data, where the X-ray structure is known, has been used for analysing conformational changes. ${ }^{23}$ With GroEL, a chaperone responsible for protein folding, the correspondence between X-ray structure and single particle cryoEM reconstruction has been achieved at the level of $\sim 11 \AA$ resolution. ${ }^{24}$ Moreover, functionally ATP-bound states of GroEL have been solved in the sub-10 $\AA$ range using cryoEM images. ${ }^{25}$

Here we report the structure of the $1 \times 6$-mer hemocyanin of the European spiny lobster Palinurus elephas at a resolution of $8 \AA$ from cryoEM data. This structure has been compared with the known X-ray structure of the hemocyanin of the closely related species $P$. interruptus.

\section{Results}

Primary structure of $\boldsymbol{P}$. elephas hemocyanin (PelHc)

Three slightly different hemocyanin subunit sequences were obtained from a hepatopancreas cDNA library of P. elephas (named PelHc1 to 


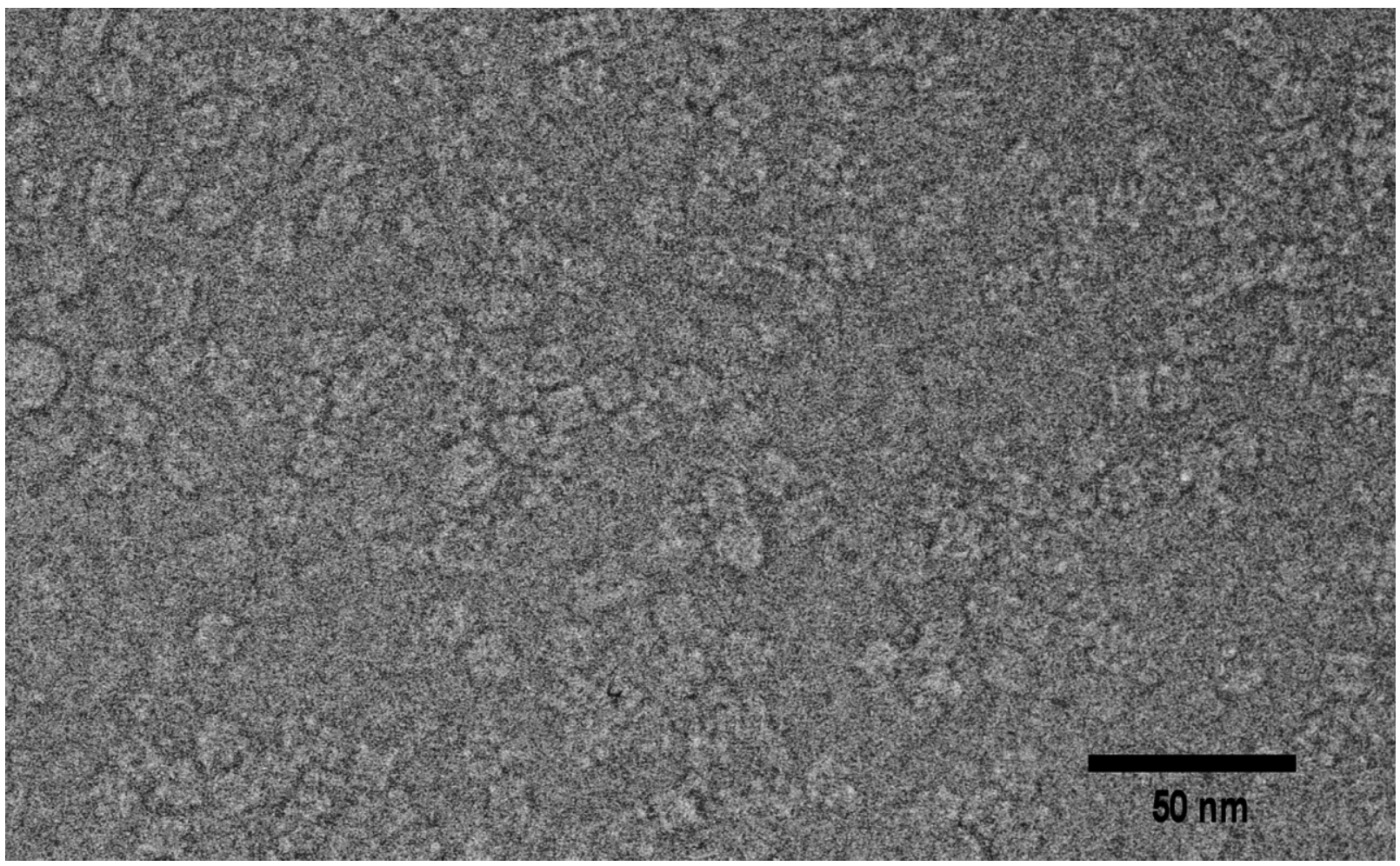

Figure 2. A representative cryo-electron micrograph of PelHc. The $1 \times 6$-mer hemocyanin molecules are visible at low contrast in the vitreous ice near to focus.

PelHc3 hereinafter; EMBL/GenBank accession numbers AJ344361-AJ344363). The deduced proteins contain 656 (PelHc1 and PelHc2) and 657 (PelHc3) amino acid residues, excluding the signal peptides (Figure 1). The three P. elephas subunits differ at 25-38 amino acid positions (94.2-96.2\% identity; $97.0-98.3 \%$ similarity considering isofunctional amino acid replacements). A single amino acid residue insertion was observed in PelHc3 between $\beta$-sheets 3D and 3E. Comparison of three PelHc sequences with the two hemocyanin subunits $a$ and $b$ of P. interruptus hemocyanin (PinHca, PinHc $b)^{26,27}$ shows that the sequences of the two species differ at 119-131 amino acid positions (80.0-81.9\% identity; 91.6-92.4\% similarity) (Figure 1). Phylogenetic analyses show that all three of the P. elephas subunits and the $P$. interruptus $a$ and $b$ subunits belong to the $\alpha$-type crustacean hemocyanin sub-family. ${ }^{4,9,28}$

Table 1. Distribution of single particle in micrographs and evaluated defocus

\begin{tabular}{lcc}
\hline Micrograph & Number of images & Defocus $(\mu \mathrm{m})$ \\
\hline 1 & 1203 & 1.6 \\
2 & 3712 & 1.9 \\
3 & 1477 & 2.5 \\
4 & 748 & 2.6 \\
5 & 1830 & 2.6 \\
6 & 1000 & 2.9 \\
\hline
\end{tabular}

\section{Cryoelectron microscopy and 3D-analysis}

Hemocyanin was purified from the hemolymph of P. elephas by size-exclusion chromatography. The purity of the samples was assessed by native PAGE, SDS-PAGE and negative staining EM. Cryoelectron micrographs were recorded as described in Materials and Methods. A total of $99701 \times 6$-mer PelHc particles were interactively selected from six electron micrographs (Figure 2). Defocus was evaluated for each negative and results are shown in Table 1 . Comparison of the nominal instrumental defocus with that determined from the micrographs demonstrated that it was systematically shifted by $\sim 0.9 \mu \mathrm{m}$. Images from each micrograph were individually corrected for the phase contrast transfer function (CTF).

A reference-free alignment (RFA) was performed for 5258 molecules extracted from two electron micrographs. The following classification by multi-variate statistical analysis (MSA) enabled us to obtain a first set of references for multi-reference alignment (MRA) and then to extract the first 20 classes for 3D analysis, including several top views, but mainly side views; top: $\beta$-angle $=0^{\circ}$, side: $\beta$-angle $=90^{\circ}$; see Figure $3(\mathrm{~b})$ ). Re-projections of the first reconstruction were used for the next round of MRA and MSA. Four iterations were performed, including both alignment with classification and determination of angular orientation. During the refinement the number of Hc particles was progressively increased, to the 

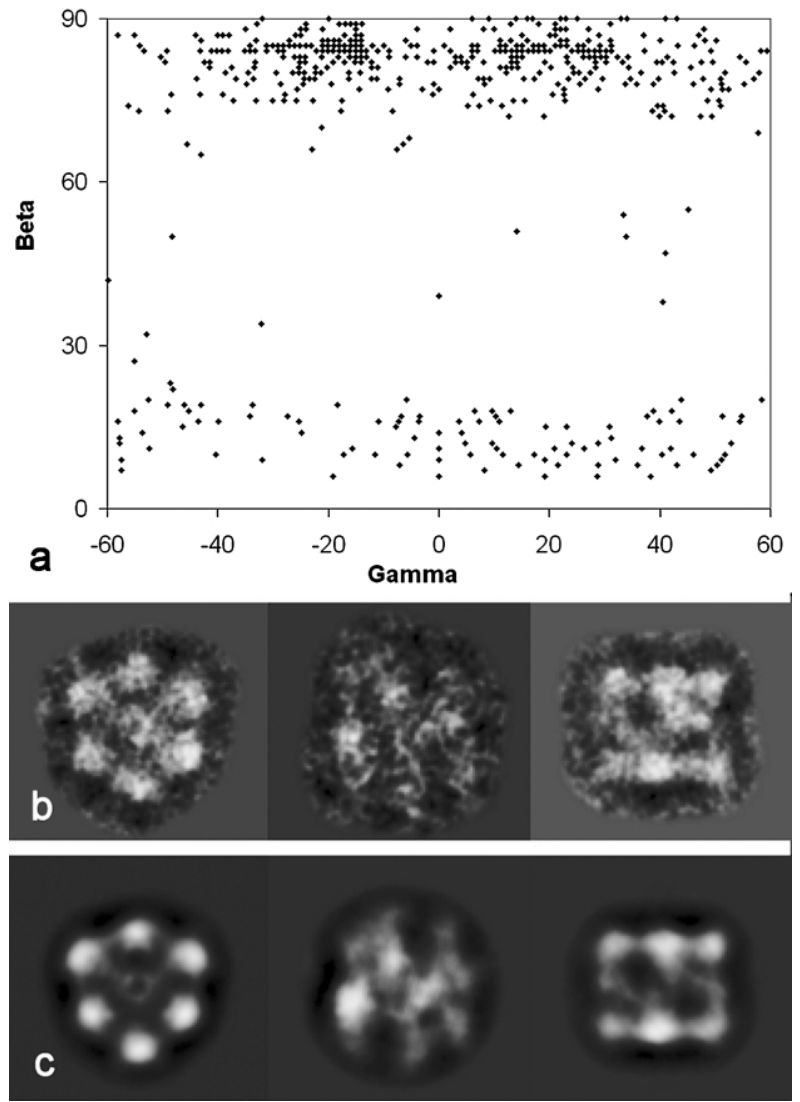

Figure 3. A $3 \mathrm{D}$ reconstruction of the spiny lobster P. elephas hemocyanin hexamers from cryoEM. (a) The distribution of the Euler-angles within the data set, (b) class average and (c) corresponding reprojection of a top, a tilt and a side view, are shown.

final figure of 9970 , to achieve higher resolution. Classification of the complete set resulted in $\sim 1000$ class averages. The molecule revealed two preferred orientations: side views with $\beta$-angle close to $90^{\circ}$ and top views with $\beta$-angle close to $0^{\circ}$. Therefore, to avoid over-weighting the analysis with top views, in the final reconstruction their number was reduced to about $10 \%$. Eventually, the 3D reconstruction was obtained from 600 classes. The distribution of Euler angles of the classes is shown in Figure 3(a). The distribution reveals side views of the molecule at different $\gamma$ angles and several tilted views; this resembles the coaxial angular distribution obtained for keyhole limpet hemocyanin, and is almost what is required for a full tomographical reconstruction. ${ }^{29}$ Examples for each class type (top view, tilted view and side view) are presented in Figure 3(b), with reprojections in Figure 3(c).

The final density map of PelHc (1 $\times 6$-mer) demonstrates the detailed structure, as illustrated by the sections through the molecule (Figure 4(a)). The FSC curve crosses the $6 \times 3 \sigma$ curve $^{29}$ at $8.0 \mathrm{~A}$; the 0.5 half height line is crossed at about $11 \AA$ (Figure 5).

\section{Comparison of the density maps of the X-ray structure of PinHc to the 3D cryo-TEM reconstruction of PelHc}

As shown in Figure 4, the two maps, displayed as sections, gives an initial indication of the close similarity of the two structures. In the majority of the sections, a nearly perfect match can be seen. Only the sections 31-33 and 67-69 show some differences in the densities, resulting from a few amino acid residues that are not resolved in the X-ray structure of PinHc (Figure 4(b)). These residues are displayed in green in Figure 8, below, for one subunit after manual fitting inside the cryoEM density map. In addition, the densities within sections 48-51 shown in Figure 4 differ in their distribution, though they have general features in common.

\section{Fitting of the X-ray structure of PinHc into the 3D cryoEM reconstruction of PelHc}

Using the computer program MOLREP, automatic 3D fitting of the X-ray structure of PinHc $(1 \times 6$-mer) to the cryoEM density map of PelHc (Figure 6) was performed. The handedness of the oligomer was already known from the PinHc X-ray structure, therefore, the density map of PelHc was adjusted accordingly. This produced six highly significant peaks at the beginning of a series of 30 peaks, as expected for a hexameric structure. A manual approach also revealed an excellent match of the two structures, which was confirmed by the automatic cross-correlation procedure within the program MOLREP. Indeed, the result from this auto-correlation agreed very well with manual fitting (within $2^{\circ} / 2 \AA$ ).

\section{Fitting of the homology model of the PelHc monomer into the cryoEM reconstruction}

The modelled structure of the PelHc monomer was fitted for the six masses within the D3 symmetric molecule in the EM density map, within MOLREP, using the six highest correlation peaks. Within the density map the modelled PelHc $(1 \times 6)$ hexamer fills all available mass (Figure 7(a) and (b)). Furthermore, it is shown that secondary structure elements, such as single $\alpha$-helices, correlate with internal and surface structural features of the cryoEM 3D reconstruction. The individual monomers of PelHc, which were modelled as described above for direct comparison with the X-ray data of PinHc (Figure 7(c)), show a high correlation. It should be emphasized that both of these quaternary structures were created independently.

\section{Discussion}

A 3D reconstruction of biological molecules using cryoEM data is a representative and independent 


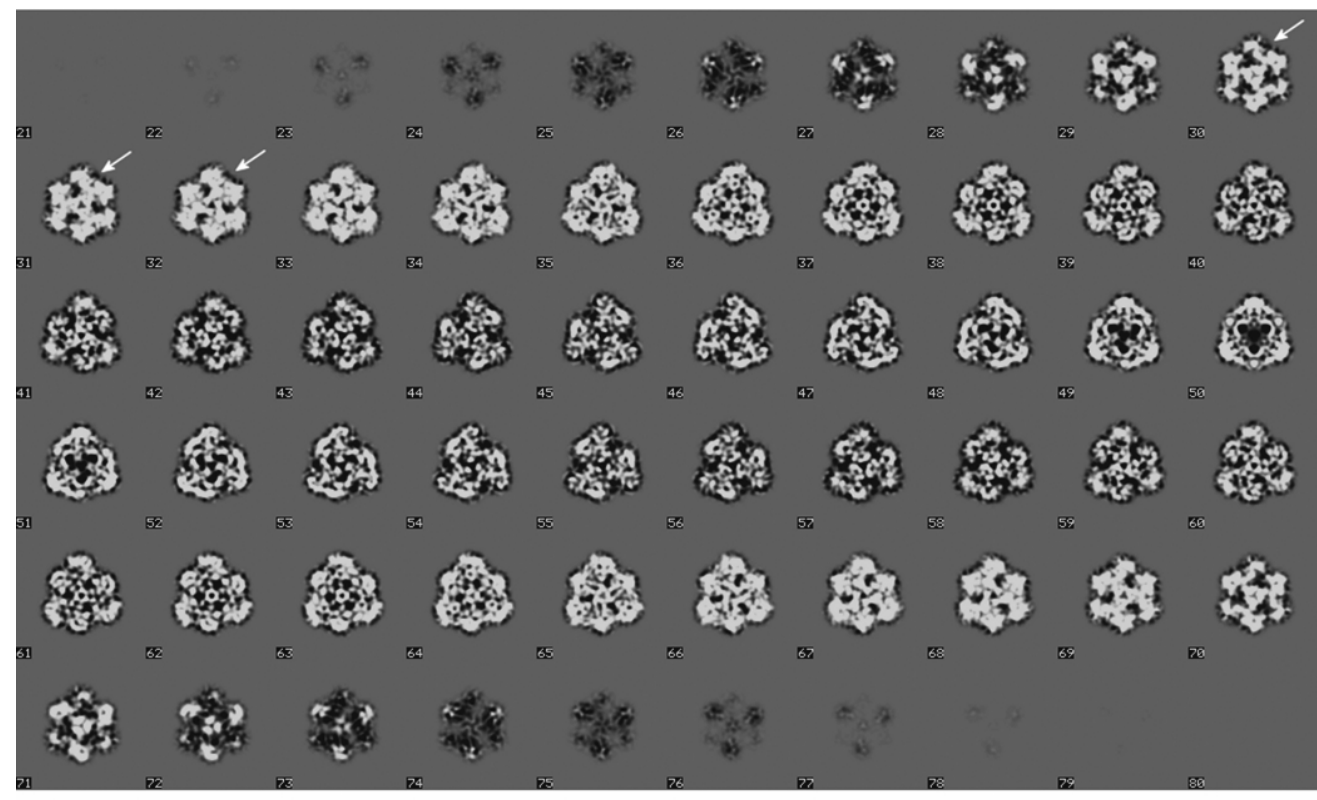

a

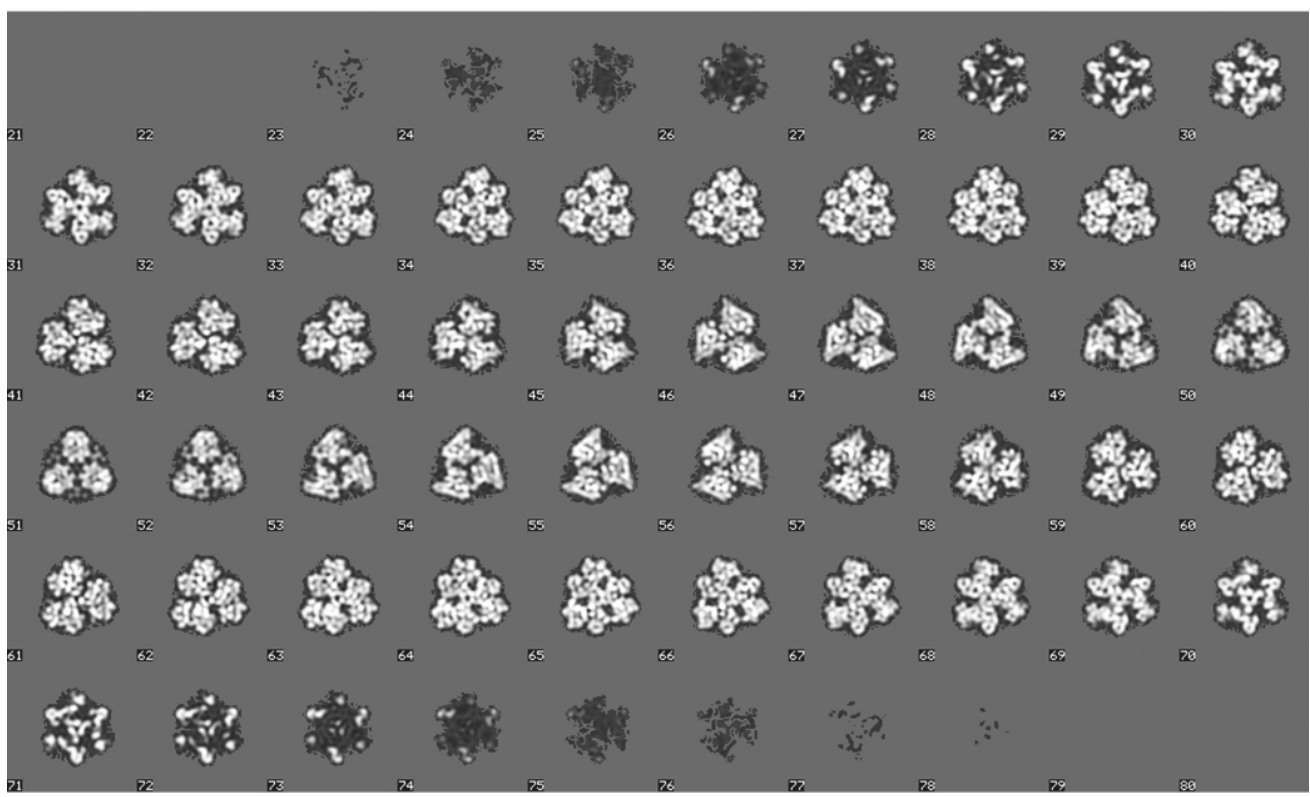

b

Figure 4. Equivalence of the tomographic map of the two lobster hemocyanins. (a) Sections through the density map of native PelHc and (b) the X-ray structure of the re-associated PinHc subunit $a$ hexamer show the same general shape. Additional density can be detected in the EM density map because a few amino acid residues are not included in the X-ray structure (arrows).

method that allows the study of structures under essentially physiological fully hydrated conditions. We present here the first high-resolution structure $(8 \AA)$ of an arthropod hemocyanin $1 \times 6$-mer obtained from cryoEM, and correlation with X-ray data from a highly homologous molecule.

\section{Quality of the sequence alignment}

The $a$ and $b$ subunits of PinHc are closely related $(96.5 \% \text { sequence identity })^{27}$ and belong to the $\alpha$-type of crustacean hemocyanin subunits as defined immunologically ${ }^{4}$ and confirmed by sequence analyses., ${ }^{9,10}$ The native molecule contains a third subunit $c(\sim 16 \%)$, which differs in $\sim 40 \%$ of its amino acid residues and is a $\gamma$-type hemocyanin subunit. ${ }^{4,30}$ It should be emphasised that Gaykema et $a .^{11}$ used a $1 \times 6$-mer artificial reassembly product from the two purified PinHc $\alpha$-type subunits $(a$ and $b$ ) for their X-ray crystallographic study. To avoid structural deviations caused by the presence of a $\gamma$-type subunit, possible structural damage during subunit isolation and reassembly artefacts, we have preferred the use of native PelHc for our cryoEM study, as this protein contains only $\alpha$-type subunits. ${ }^{4}$ Nevertheless, P. elephas 


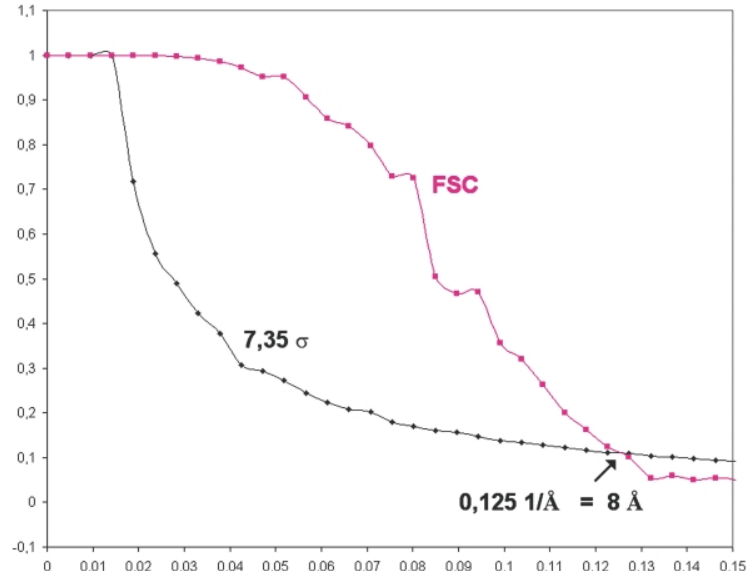

Figure 5. Determination of the resolution. The FSC curve crosses the $6 \times 3 \sigma$ curve at $8.0 \AA$. (Determination of the resolution using the 0.5 criterion shows that the half-height line is crossed at $\sim 11 \AA$.)

(the European spiny lobster) and P. interruptus (the American spiny lobster) are closely related species and no major differences are to be expected between the amino acid sequences of their $\alpha$-type hemocyanin subunits. In fact, the three PelHc $\alpha$-type sequences share about $80-82 \%$ amino acid identity with PinHc subunits $a$ and $b$, and are about $92 \%$ similar when iso-functional substitutions are considered (according to a PAM250 matrix). Intra-specific variations within PelHc are minimal and only account for about $3.8-5.8 \%$ of the amino acid difference among subunits. Thus, only a minor alteration of the cryoEM 3D reconstruction is expected due to unknown or randomly positioned amino acid residues within the subunits in the native PelHc hexamer.

\section{Molecular modelling}

PelHc and PinHc display a high degree of sequence similarity $(>80 \%$, identity), which allows the modelling of PelHc by utilizing the known
PinHc structure. However, the published PinHc $X$-ray structure does not include amino acid residues 596-605 and 654-657 (cf. Figure 1). In general, molecular modelling of tertiary structure is not simple if one has large differences in the amino acid sequence. However, the high homology of the PelHc subunit 1 and PinHc subunit $a$ greatly facilitated the process. After sequence alignment, the absence of residues 596-605 in the PinHc subunit $a$ is directly indicated (displayed green in Figure 8). These residues were initially arranged for PelHc subunit 1 as an outgoing loop by the automated "magic fit" algorithm in SWISSMODEL. This provided the conditions we needed to model the loop residues manually into the density map. The fact that these residues were not resolved in the X-ray structure of PinHc indicates that there was a region of high disorder in the X-ray data. However, the available mass in the EM density map correlates with the location of these absent PinHc amino acid residues. The modelling (Figure 8), using the EM density as reference, is highly persuasive as no other reasonable location of these amino acid residues is possible.

\section{Conclusions and future perspectives}

With a resolution of $8 \AA$, the $10 \AA$ barrier has now been passed for the $3 \mathrm{D}$ reconstruction of an arthropod hemocyanin by cryoEM. In the case of highly symmetric particles, such as viruses, resolutions better than $6 \AA$ have been achieved, but molecules with simple symmetries such as the D3 symmetry of the PelHc, a data set of many thousand single molecule images is needed to reach high resolution. We have achieved this criterion by the selection of almost 10,000 particles for the image analysis, of which $\sim 6000$ (within 600 classes) contributed the final 3D reconstruction.

Molecular fitting/docking has increasingly become a standard method used for comparison of molecular details from X-ray structural analysis with cryoEM 3D reconstructions. For some structures, such as viruses and chaperones, X-ray and

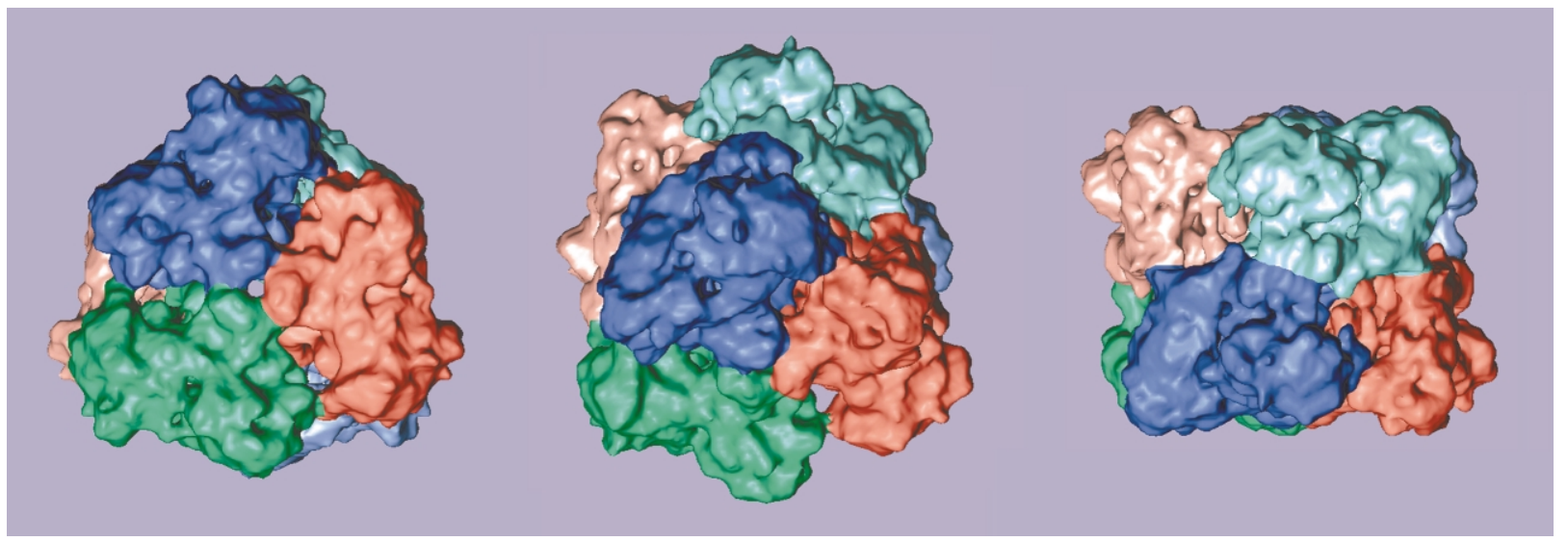

Figure 6. A 3D-reconstruction of PelHc at $8 \AA$ resolution. The single subunits of the hexamer are highlighted by using different colours (red, green and blue). 

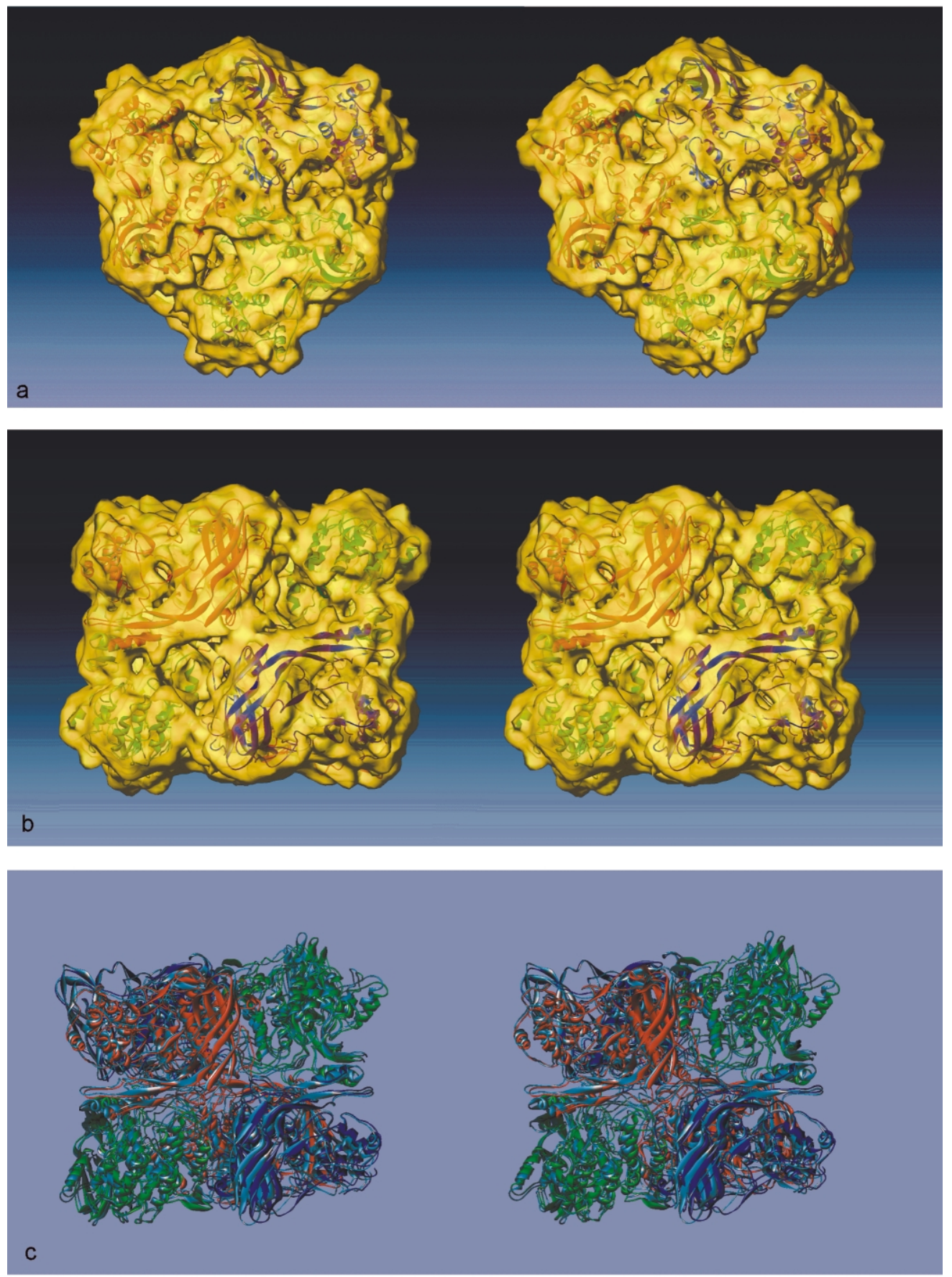

Figure 7. Stereo views of the six automatically fitted PelHc1 subunits into a Hc hexamer using the PelHc cryoEM 3D reconstruction as reference. The upper part shows (a) top and (b) side views of the hexamer with fitted subunits. The subunits are coloured red, green and blue, the cryoEM density map, used as reference, is in transparent gold. (c) In the lower part, the re-associated PinHc a subunit hexamer from X-ray data is shown in uniform light blue, superimposed by six subunits (red, green and blue) of the modelled PelHc1 sequences, fitted as in the cryoEM 3D reconstruction.

EM data have been directly compared..$^{25,31}$ In the case of our crustacean hemocyanin 3D reconstruction, we also show that parts of the molecule that are absent in the X-ray structure can be modelled within the EM density map from the available amino acid sequence. The comparison of the closely related hemocyanins, PinHc and PelHc, has shown an extremely high correlation for structural features (Figure 7(c)). This approach shows that using the subunit structure that is already known for a closely related protein, the highresolution structure of an oligomer can be solved from the cryoEM 3D reconstruction of the oligomer and the available amino acid sequence.

The 3D reconstruction of PelHc described here does not enable us to assess the level of oxygenation of the molecule at the time of specimen freezing. Although it is likely that the molecule 

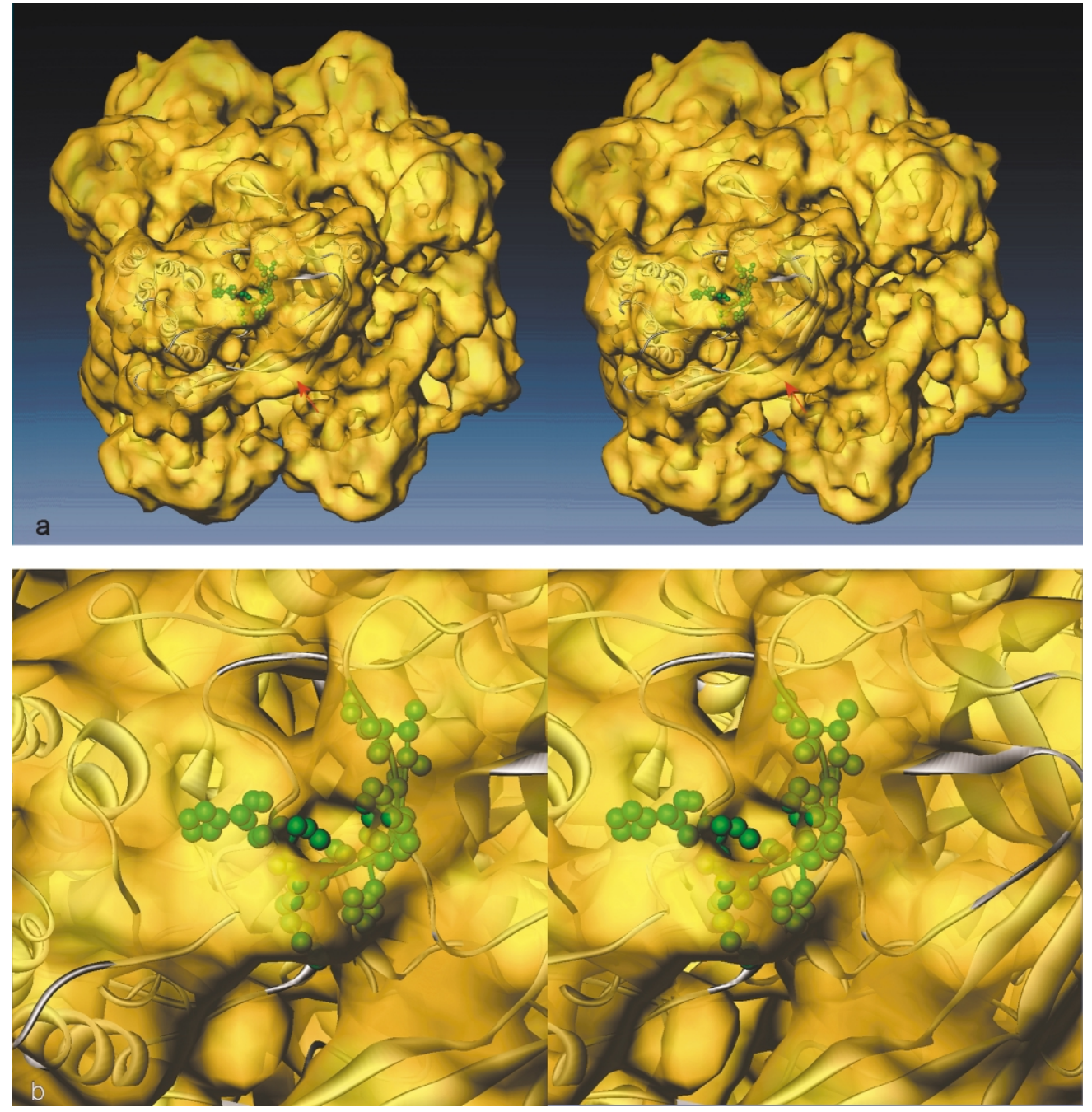

Figure 8. Stereo view of a PelHc1 subunit model fitted into the cryoEM PelHc EM density map. (a) The amino acid sequence of PelHc1 used for modelling the 3D structure of the monomer is shown as a silver ribbon. The red arrow denotes the four amino acid residues at the $\mathrm{C}$ terminus, missing in the PinHca X-ray structure. (b) Residues (596-605) that are not resolved in the X-ray structure of PinHca, are modelled in PelHc, and shown in green.

was initially fully oxygenated, the rapid fall through the nitrogen atmosphere during plunge freezing may have produced some deoxygenation. Assuming that the arrangement of the subunits within the hexamer changes during deoxygenation, as described by de Haas et al., ${ }^{32}$ there is a high degree of similarity of the quaternary structure of our PelHc reconstruction with the X-ray data of PinHc ${ }^{11}$ (obtained using oxygenated hemocyanin). This led us to the likely conclusion that we had prepared oxygenated hemocyanin.

Nevertheless, if in future controlled oxygenation/deoxygenation can be achieved during the freezing process, the possibility remains that $3 \mathrm{D}$ reconstructions from cryoEM may have sufficient resolution to reveal structural changes. The technical difficulties are by no means trivial in case of the oxygenated form, but deoxygenated hemocyanin may be more readily produced by using a nitrogen atmosphere before sample preparation. Our 3D reconstruction of the PelHc $1 \times 6$-mer also has potential as an internal control for our on- going studies aimed at the $3 \mathrm{D}$ reconstruction of related protein molecules (for which X-ray structures are not currently available) from cryoEM images. These are the insect hexamerins, ${ }^{33,34}$ the arthropod phenoloxidases ${ }^{35}$ and a number of different arthropod multi-hexamers. ${ }^{1}$

\section{Materials and methods}

\section{cDNA sequencing and analyses}

Total RNA was extracted from the hepatopancreas of P. elephas using the guanidinium thiocyanate method. ${ }^{36}$ The poly $(\mathrm{A})^{+}$RNA was purified using the PolyATract kit (Promega). A directionally cloned cDNA expression library was constructed using the Lambda ZAP-cDNA synthesis kit (Stratagene). The library was amplified once and screened with $P$. interruptus hemocyanin $\alpha$-subunit antibodies (kindly provided by $\mathrm{H}$. Decker \& J. J. Beintema). Hemocyanin-positive phage clones were converted to plasmid vectors using the reagents provided by Stratagene and sequenced on both strands by 
a commercial service (Genterprise, Mainz, Germany). The Genetics Computer Group (GCG) sequence analysis software package version 8.0 and the tools provided by the ExPASy Molecular Biology Server of the Swiss Institute of Bioinformatics $\dagger$ were used for sequence analyses. The P. elephas hemocyanin (PelHc) sequences were manually aligned with a previously published alignment of the arthropod hemocyanin superfamily., ${ }^{9,28}$

\section{Hemocyanin purification}

The hemolymph was withdrawn from the abdominal sinus of a living adult $P$. elephas with a syringe and centrifuged for ten minutes at $10,000 \mathrm{~g}$. The cell-free supernatant was applied to a Biogel A5m (BioRad) column $(1.5 \mathrm{~cm} \times 100 \mathrm{~cm})$. Elution was performed at $4{ }^{\circ} \mathrm{C}$ with $100 \mathrm{mM}$ Tris- $\mathrm{HCl}$ (pH 7.5), $10 \mathrm{mM} \mathrm{MgCl}, 5 \mathrm{mM} \mathrm{CaCl}_{2}$. The purity of the eluted material (PelHc) was analysed by SDS-PAGE and native PAGE.

\section{Preparation and processing of cryoelectron microscopy}

Initially, the purified hemocyanin was assessed by negative stain EM and then analysed under cryo conditions. A droplet of the purified hemocyanin was applied to a glow-discharged holey carbon support film. Excess fluid was removed by blotting and the grid was rapidly plunge-frozen in liquid ethane. ${ }^{37}$ Cryo-transfer was performed using a Gatan model $626 \mathrm{DH}$ cryo-holder to a Philips CM200 equipped with a field emission gun (FEG) operated at an accelerating voltage of $160 \mathrm{kV}$. The underfocus was set between $1.0 \mu \mathrm{m}$ and $2.9 \mu \mathrm{m}$, at $50,000 \times$ instrumental magnification. Electron micrographs were recorded under low-dose conditions using Agfa Scienta film. Negatives were developed for 12 minutes in full-strength Kodak D19 developer.

\section{Image processing}

Electron micrographs without drift and astigmatism were scanned and digitized using a TANGO drum scanner (Heidelberger Druckmaschinen, Heidelberg, Germany) with $10.6 \mu \mathrm{m}$ step size corresponding to $2.12 \AA$ on the negative. Image processing was performed using the IMAGIC-5 software package. ${ }^{22,38}$ Images of single particles were selected interactively from negatives and extracted within $256 \times 256$ pixel boxes. Defocus of each micrograph was evaluated from positions of zeros of phase CTF on incoherently averaged Fourier spectra $^{39}$ of $\sim 200$ randomly selected images. To reduce the background and make positions of CTF zeros more clear in the averaged spectra they were band-pass filtered. Correction of CTF was done by flipping phases of even rings. For the further analysis, images were normalized and low frequencies suppressed using a high-pass filter. Centring of the images was done using a $\mathrm{RFA}^{40}$ and that was followed by $\mathrm{MSA}^{38,41}$ to elucidate characteristic views of the molecule. Then refinement of class averages, presenting reliable views of the molecule, was performed with the iterative procedure of MRA and classification by MSA. To find relative orientations of class averages angular reconstitution was used, ${ }^{42}$ with imposed D3 symmetry. The 3D reconstructions were calculated using the exact filter back-projection

$\dagger$ http:/ / www.expasy.org approach..$^{43}$ The best classes were used to calculate a 3D map and then to refine angular orientation for all classes.22,38,44 Resolution was determined by Fourier shell correlation (FSC $)^{43}$ by comparing two reconstructions, each calculated from half of the data set. The final density map was filtered to nominal $7.5 \AA$, so that structural details of a size of $(\leq 7 \AA$ were removed. For further processing, the $3 \mathrm{D}$ map was converted to several data formats. Usually, protein data from X-ray databases was compared with density maps from 3D EM within the CCP4 file format (for MOLREP). The CCP4 and IMAGIC-5 file formats were used for analysis in AMIRA.

\section{Protein structure modelling and prediction of tertiary structures}

Homology models of the PelHc subunits were built using the SwissPDB-Viewer ${ }^{45,46}$ with the three-dimensional structure of PinHc (RCSB PDB-IDCODE: 1HCY REVISION-LEVEL: 1HCY), containing equal quantities of subunit A and subunit B, as a template. The structure was refined using the Swiss-Model server $+{ }^{47}$ One of the resulting models of the monomer was fitted automatically into the density map from the cryoEM 3D reconstruction using the cross-rotation function (RF) and phased transfer function (PTF) of the MOLREP Software (see below). Structural features that were absent in the $3.2 \AA$ X-ray structure of PinHc (residues 596-605) were then manually fitted into the density map of the PelHc reconstruction using the Swiss PDB-Viewer. For the four C-terminal amino acid residues (654-657), a mass correlation is recognizable (Figure 8). However, in this case the exact positions of the amino acid residues could not determined with sufficient confidence.

\section{Fitting of three-dimensional structures}

Initially, to test the similarity the structure, the PinHc $(1 \times 6)$ crystal structure was fitted into the cryoEM density map of the hemocyanin by two steps. Firstly, a manual fit enabled the positioning of the PinHc hexamer within the EM density map using the program O. ${ }^{48}$ In a second step, the molecular fit was refined automatically. The PinHc hexamer was thereby accurately fitted into the cryoEM density of PelHc. The RF was calculated at 212.0-6.1 $\AA$, using a radius of integration of $95.4 \AA$. The integration radius is calculated from twice of radius gyration of the atomic model. The maximum RF is defined by unit cell parameters (box size of the cryoEM density); the minimum RF resolution depends on the radius of integration. ${ }^{49}$

To produce a model of the PelHc $(1 \times 6$-mer $)$ quaternary structure at atomic resolution the modelled PelHc1 subunit (see above) was applied six times as a search model for molecular replacement into the cryoEM density map of the 3D reconstruction. The search was performed using MOLREP. The PTF and the $\mathrm{RF}^{50,51}$ were calculated for each monomer, and the six highest peaks of correlation within the cryoEM map were used for determination of the spatial orientation of the monomers. In this case, the RF was then calculated at 212.0-3.2 $\AA$, using a radius of integration of $50.2 \AA$. For visualisation WEBLABVIEWER PRO (Accelrys $\mathrm{GmbH}$, München, Germany) was used to create a VRML File that could readily be compared with the cryoEM map within AMIRA 2.3 (TGS Europe, Düsseldorf, Germany). 


\section{Acknowledgements}

We thank Jaap J. Beintema and Heinz Decker for the antibodies, Anne Hembach and Cornelia Milke for their help in performing the cDNA cloning, Michail Isupov and Alexei Vagin for the program MOLREP and Bruno Klaholz for introduction to "O" and the rigid-body-fit program, which were used for preliminary fitting studies. This work has been supported by grants from the DFG (Ma843/ 4-3, Bu956/3-5) and the Stiftung Innovation von Rheinland-Pfalz (GZ. 8312-386 261/281).

\section{References}

1. Markl, J. \& Decker, H. (1992). Molecular structure of the arthropod hemocyanins. In Advances in Comparative and Environmental Physiology (Mangum, C. P., ed.), vol. 13, pp. 325-376, Springer, Berlin.

2. van Holde, K. E. \& Miller, K. I. (1995). Hemocyanins Advances in Protein Chemistry, vol. 47, Academic Press, San Diego pp. 1-81.

3. Meissner, U., Dube, P., Harris, J. R., Stark, H. \& Markl, J. (2000). Structure of a molluscan hemocyanin didecamer ( $\mathrm{HtH} 1$ from Haliotis tuberculata) at $12 \AA$ resolution by cryoelectron microscopy. J. Mol. Biol. 298, 21-34.

4. Markl, J., Stöcker, W., Runzler, R. \& Precht, E. (1986). Immunological correspondences between the hemocyanin subunits of 86 arthropods: evolution of a multigene Protein family. In Invertebrate Oxigen Carriers (Linzen, B., ed.), pp. 281-292, Springer, Berlin.

5. Savel-Niemann, A., Markl, J. \& Linzen, B. (1988). Hemocyanins in spiders XXII. Range of allosteric interaction in a four-hexamer hemocyanin. Cooperativity and Bohr effect in dissociation intermediates. J. Mol. Biol. 204, 385-395.

6. Robert, C. H., Decker, H., Richey, B., Gill, S. J. \& Wyman, J. (1987). Nesting: hierarchies of allosteric interactions. Proc. Natl Acad. Sci. USA, 84, 1891-1895.

7. Leidescher, T. \& Decker, H. (1990). Conformational changes of tarantula (Eurypelma californicum) hemocyanin detected with a fluorescent probe, 7-chloro-4nitrobenzo-2-oxa-1,3-diazole. Eur. J. Biochem. 187, 617-625.

8. Sterner, R., Bardehle, K., Paul, R. \& Decker, H. (1994). Tris-an allosteric effector of tarantula hemocyanin. FEBS Letters, 339, 37-39.

9. Burmester, T. (2001). Molecular evolution of the arthropod hemocyanin superfamily. Mol. Biol. Evol. 18, 184-195.

10. Burmester, T. (2002). Origin and evolution of arthropod hemocyanins and related proteins. J. Comp. Physiol. [B], 172, 95-117.

11. Gaykema, W. P., Hol, W. G., Vereijken, J. M., Soeter, N. M., Bak, H. J. \& Beintema, J. J. (1984). $3.2 \AA$ structure of the copper-containing, oxygen-carrying protein Panulirus interruptus hemocyanin. Nature, $309,23-29$

12. Hazes, B., Magnus, K. A., Bonaventura, C., Bonaventura, J., Dauter, Z., Kalk, K. H. \& Hol, W. G. (1993). Crystal structure of deoxygenated Limulus polyphemus subunit II hemocyanin at $2.18 \AA$ resolution: clues for a mechanism for allosteric regulation. Protein Sci. 2, 597-619.
13. Gaykema, W. P., Volbeda, A. \& Hol, W. G. (1986). Structure determination of Panulirus interruptus hemocyanin at $3.2 \AA$ resolution. Successful phase extension by sixfold density averaging. J. Mol. Biol. 187, 255-275.

14. Volbeda, A. \& Hol, W. G. (1989). Crystal structure of hexameric hemocyanin from Panulirus interruptus refined at $3.2 \AA$ resolution. J. Mol. Biol. 209, 249-279.

15. Boisset, N., Radermacher, M., Grassucci, R., Taveau, J. C., Liu, W. P., Lamy, J. et al. (1993). 3-Dimensional immunoelectron microscopy of scorpion hemocyanin labeled with a monoclonal Fab fragment. I. Struct. Biol. 111, 234-244.

16. Boisset, N., Penczek, P., Taveau, J. C., Lamy, J. \& Frank, J. (1995). Three-dimensional reconstruction of Androctonus australis hemocyanin labeled with a monoclonal Fab fragment. J. Struct. Biol. 115, 16-29.

17. de Haas, F., Bijlholt, M. \& van Bruggen, E. F. (1991). An electron microscopic study of two-hexameric hemocyanins from the crab Cancer pagurus and the tarantula Eurypelma californicum: determination of their quaternary structure using image processing and simulation models based on X-ray diffraction data. J. Struct. Biol. 107, 86-94.

18. de Haas, F. (1993). Electron microscopic studies on structure and function of arthropod hemocyanins. Thesis. University of Groningen.

19. Taveau, J. C., Boisset, N., Lamy, J., Lambert, O. \& Lamy, J. N. (1997). Three-dimensional reconstruction of Limulus polyphemus hemocyanin from cryoelectron microscopy. J. Mol. Biol. 266, 1002-1015.

20. van Heel, M. \& Dube, P. (1994). Quaternary structure of multihexameric arthropod hemocyanins. Micron, 25, 387-418.

21. Boisset, N., Taveau, J. C. \& Lamy, J. N. (1990). An approach to the architecture of Scutigera coleoptrata hemocyanin by electron microscopy and image processing. Biol. Cell, 68, 73-84.

22. van Heel, M., Gowen, B., Matadeen, R., Orlova, E. V., Finn, R., Pape, T. et al. (2000). Single-particle electron cryo-microscopy: towards atomic resolution. Quart. Rev. Biophys. 33, 307-369.

23. Harris, J. R., Schroder, E., Isupov, M. N., Scheffler, D., Kristensen, P., Littlechild, J. A. et al. (2001). Comparison of the decameric structure of peroxiredoxin-II by transmission electron microscopy and X-ray crystallography. Biochim. Biophys. Acta, 1547, 221-234.

24. Ludtke, S. J., Jakana, J., Song, J. L., Chuang, D. T. \& Chiu, W. (2001). A $11.5 \AA$ single particle reconstruction of GroEL using EMAN. J. Mol. Biol. 314, 253-262.

25. Ranson, N. A., Farr, G. W., Roseman, A. M., Gowen, B., Fenton, W. A., Horwich, A. L. \& Saibil, H. R. (2001). ATP-bound states of GroEL captured by cryo-electron microscopy. Cell, 107, 869-879.

26. Bak, H. J. \& Beintema, J. J. (1987). Panulirus interruptus hemocyanin. The elucidation of the complete amino acid sequence of subunit a. FEBS Letters, 204, 141-144.

27. Jekel, P. A., Bak, H. J., Soeter, N. M., Vereijken, J. M. \& Beintema, J. J. (1988). Panulirus interruptus hemocyanin. The amino acid sequence of subunit $b$ and anomalous behaviour of subunits $a$ and $b$ on polyacrylamide gel electrophoresis in the presence of SDS. Eur. J. Biochem. 178, 403-412.

28. Kusche, K. \& Burmester, T. (2001). Molecular cloning and evolution of lobster hemocyanin. Biochem. Biophys. Res. Commun. 282, 887-892. 
29. Orlova, E. V., Dube, P., Harris, J. R., Beckman, E., Zemlin, F., Markl, J. \& van Heel, M. (1997). Structure of keyhole limpet hemocyanin type 1 (KLH1) at $15 \AA$ resolution by electron cryo-microscopy and angular reconstitution. J. Mol. Biol. 271, 417-437.

30. Neuteboom, B., Jekel, P. A. \& Beintema, J. J. (1992). Primary structure of hemocyanin subunit $c$ from Panulirus interruptus. Eur. J. Biochem. 206, 243-249.

31. Grimes, J. M., Fuller, S. D. \& Stewart, D. I. (1999). Complementing crystallography: the role of cryoelectron microscopy in structural biology. Acta Crystallog. sect. D, 55, 1742-1749.

32. de Haas, F., van Breemen, J. F., Boekema, E. J., Keegstra, W. \& van Bruggen, E. F. (1993). Comperative electron microscopy and image analysis of oxy- and deoxy-hemocyanin from the spiny lobster Panulirus interruptus. Ultramicroscopy, 49, 426-435.

33. Burmester, T. (1999). Evolution and function of the insect hexamerins. Eur. J. Entomol. 96, 213-225.

34. Telfer, W. H. \& Kunkel, J. G. (1991). The function and evolution of insect storage hexamers. Annu. Rev. Entomol. 36, 205-228.

35. Decker, H., Ryan, M., Jaenicke, E. \& Terwilliger, N. (2001). SDS-induced phenoloxidase activity of hemocyanins from Limulus polyphemus, Eurypelma californicum, and Cancer magister. J. Biol. Chem. 276, 17796-17799.

36. Chirgwin, J. M., Przbyla, A. E., MacDonald, R. J. \& Rutter, W. J. (1979). Isolation of biologically active ribonucleic acid from sources enriched in ribonuclease. Biochemistry, 18, 5294.

37. Adrian, M., Dubrochet, J., Lepault, J. \& McDowall, A. W. (1984). Cryo-electron microscopy of viruses. Nature, 308, 32-36.

38. van Heel, M., Harauz, G., Orlova, E. V., Schmidt, R. \& Schatz, M. (1996). A new generation of the IMAGIC image processing system. J. Struct. Biol. $116,17-24$

39. Zhou, Z. H., Hardt, S., Wang, B., Sherman, M. B., Jakana, J. \& Chiu, W. (1996). CTF determination of images of ice-embedded single particles using a graphics interface. J. Struct. Biol. 116, 216-222.

40. Dube, P., Tavares, P., Lurz, R. \& van Heel, M. (1993). The portal protein of bacteriophage SPP1: a DNA pump with 13-fold symmetry. EMBO J. 12, 1303-1309.

41. van Heel, M., Winkler, H., Orlova, E. \& Schatz, M. (1992). Structure analysis of ice-embedded single particles. Scanning Microsc. Suppl. 6, 23-42.

42. Serysheva, I. I., Orlova, E. V., Chiu, W., Sherman, M. B., Hamilton, S. L. \& van Heel, M. (1995). Electron cryomicroscopy and angular reconstitution used to visualize the skeletal muscle calcium release channel. Nature Struct. Biol. 2, 18-24.

43. Harauz, G., Borland, L., Bahr, G. F., Zeitler, E. \& van Heel, M. (1987). Three-dimensional reconstruction of a human metaphase chromosome from electron micrographs. Chromosoma, 95, 366-374.

44. Orlova, E. V. (2000). Structural analysis of noncrystalline macromolecules: the ribosome. Acta Crystallog. sect. D, 56, 1253-1258.

45. Peitsch, M. C. (1996). ProMod and Swiss-Model: Internet-based tools for automated comparative protein modelling. J. Struct. Biol. 136, 190-200.

46. Guex, N. \& Peitsch, M. C. (1997). SWISS-MODEL and the Swiss-Pdb Viewer: an environment for comparative protein modelling. Electrophoresis, 18, 2714-2723.

47. Peitsch, M. C. (1995). Protein modelling by E-mail. Bio/Technology, 13, 658-660.

48. Jones, T. A., Zou, J. Y., Cowan, S. W. \& Kjeldgaard, M. (1991). Improved methods for building protein models in electron density maps and the location of errors in these models. Acta Crystallog. sect. A, 47, 110-119.

49. Vagin, A. A. \& Isupov, M. N. (2001). Spherically averaged phased translation function and its application to the search for molecules and fragments in electron-density maps. Acta Crystallog., sect. D, 57, 1451-1456.

50. Crowther, R. A. (1972). The fast rotation function. In The Molecular Replacement Method (Rossmann, M. G., ed.), pp. 173-178, Gordon \& Breach, New York.

51. Bentley, G. A. (1997). Phase transfer function. Methods Enzymol. 276, 611-619.

52. Linzen, B., Soeter, N. M., Riggs, A. F., Schneider, H. J., Schartau, W., Moore, M. D. et al. (1985). The structure of arthropod hemocyanins. Science, 229, 519-524.

Edited by W. Baumeister 